\title{
Self-medication and self-treatment with short-term antibiotics in Asian countries: A literature review
}

\author{
I Gusti Ayu Rai Widowati ${ }^{1}$ (D) Ni Nyoman Sri Budayanti ${ }^{2}$ iD, Pande Putu Januraga ${ }^{3}$ (D) Dyah $^{2}$ \\ Pradnyaparamita Duarsa ${ }^{4}$ iD \\ ${ }^{1}$ Doctoral Study Program, Faculty of Medicine, Udayana University, Bali, Indonesia \\ 2Department of Microbiology, Faculty of Medicine, Udayana University, Bali, Indonesia \\ ${ }^{3}$ Center of Public Health Innovation, Faculty of Medicine, Udayana University, Bali, Indonesia \\ ${ }^{4}$ Department of Public Health and Preventive Medicine, Faculty of Medicine, Udayana University, Bali, Indonesia
}

\begin{tabular}{|c|c|}
\hline $\begin{array}{l}\text { Correspondence } \\
\text { IGAR Widowati } \\
\text { Doctoral study program Faculty } \\
\text { of Medicine } \\
\text { Udayana University } \\
\text { Bali } \\
\text { Indonesia } \\
\text { gekrai@angligan.com }\end{array}$ & $\begin{array}{l}\text { Abstract } \\
\text { Introduction: The general public plays a role in the increase and spread of antimicrobial resistance } \\
\text { by seeking self-medication with antibiotics. Aim: The purpose of this systematic review is to } \\
\text { evaluate the prevalence of self-medication with short-term antibiotics in Asian countries. } \\
\text { Method: A literature search was performed on Google Scholar, PubMed, and Science Direct } \\
\text { databases in 2013-2020. Results: A total of } 36 \text { articles were included for full review and data } \\
\text { extraction. The prevalence of self-medication with antibiotics in the articles reviewed was } 50.8 \% \text {. } \\
\text { The main source of antibiotics without a prescription is from community pharmacies. The practice } \\
\text { of self-medication with antibiotics is influenced by multifactor. Conclusion: Self-medication with } \\
\text { antibiotics is a very common practice in Asian countries, contributing to the emergence of } \\
\text { antimicrobial resistance which is no longer a threat but a terrible reality. This review provides an } \\
\text { overview of the need for solutions to reduce self-medication with antibiotics behaviour in the } \\
\text { community. }\end{array}$ \\
\hline
\end{tabular}

\section{Introduction}

The world is experiencing an epidemiological transition where the trend of non-communicable diseases tends to increase along with changes in people's life behaviour. However, the problem of communicable diseases cannot be completely resolved. One of the biggest threats to global health is the uncontrolled spread of epidemics due to highly pathogenic infectious diseases, especially those that easily cross borders and have the potential to endanger communities and their economies (WHO, 2015).

Antibiotics are the most commonly purchased drugs worldwide. The overuse, underuse, or misuse of antibiotics becomes a global issue that causes negative impacts. Antimicrobial resistance (AMR) results in the wastage of scarce resources and widespread health hazards. It is very critical health, social, and economic problem worldwide. Due to the clinical and socioeconomic impacts of AMR, a strategy and action plan based on national efforts and international cooperation are needed to control and prevent AMR (Song, 2014).

Antibiotic resistance is perhaps the greatest threat facing the world in the field of infectious diseases, where it has the potential to cause greater death than cancer. The WHO report states that globally, the mortality rate due to antibiotic resistance in 2013 was $700,000 /$ year, and it is predicted that in 2050 the mortality rate due to antibiotic resistance will be $10,000,000 / y e a r$. The cumulative risk of economic expenditure is 100 billion USD/66 billion GBP. The direct and indirect impacts of antibiotic resistance will be felt in low-middle income countries in the Southeast Asia region. Every year, of the 14 million deaths that 
occur in the Southeast Asia region, six million or about $40 \%$ are caused by infectious diseases, which also contribute to $42 \%$ of the loss of disability-adjusted life years. This burden requires urgent action to tackle antibiotic resistance, mitigating economic and health costs (WHO, 2016).

Half of all drugs are inappropriately prescribed, dispensed, or sold worldwide. Medicines are often used incorrectly, where $50 \%$ of all patients fail to take the drug and adhere to its therapy rationally. Irrational use of drugs is a major problem worldwide (Fresle, Hardon $\&$ Hodgkin, 2004). Self-medicated practice refers to the use of drugs to treat complaints of self-diagnosed disease without consulting a medical practitioner and without medical supervision. Weaknesses in health care systems, especially in developing countries, such as unfair distribution, high costs, limited access, lack of professional health care, unregulated distribution of drugs, and patient attitudes towards doctors, are some of the main drivers of self-medicated behaviour (Bhatta \& Nepal, 2018).

In addition, it is widely shown that excessive use of antibiotics at the population level is an important risk factor for increased antibiotic resistance. Because patients do not know about antibiotics and are confused about their role, they must be told that most common infections do not require antibiotics and that these drugs can actually be dangerous. Antibiotic abuse that is prevalent in the community reinforces the importance of conducting research to develop effective strategies to stem the tide of antibiotic resistance (Singh, 2017). The objective of this literature search was to evaluate the prevalence and determinants of self-medication with antibiotics in Asian countries.

\section{Methods}

\section{Search strategy}

A literature search was performed on Google Scholar, PubMed, and Science Direct databases in 2013-2020. The search included a combination of "self-medication" OR "self-treatment" AND "antibiotics" AND "country where the study was conducted". The search was limited to peer-reviewed articles and reviews published in English. Search results from each database were exported to Microsoft Excel, merged, and sorted for removal of duplicate citations.

\section{Study selection}

Studies published in English are included in the review if they aim to assess the behaviour of self-medication with antibiotics in Asian countries. Studies on antiviral, antifungal, antiprotozoal, and topical antimicrobials are excluded. Original researches, research reports, case studies, and association reports were included for review. Abstract only articles, article reviews, editorials, systematic reviews, critical analysis, and narrative overviews were excluded. Articles were considered to be relevant based on the identification of self-medication with short-term antibiotics. Full-text articles were assessed for inclusion, and reasons were documented for all excluded papers.

\section{Data extraction}

Articles reporting community behaviour towards selfmedication with short-term antibiotics were selected. Differences were resolved through discussion between authors. The authors excluded long-term antibiotics from this review, such as tuberculosis and antimalarial treatment.

A standard data extraction form was used to collect the following information: authors, year of publication, the country where the study was conducted, sample size, population sampled, prevalence, disease symptoms, main reasons of behaviour, where the information was obtained, source of drugs, and choice of antibiotics.

A database search returned 192 publications for review. 148 full-text publication meets the requirements, 114 were excluded for reasons of duplicate records, review articles, irrelevant topics, time of publication, systematic review, the article is not detailed, data is not systematically displayed which did not meet the authors' research objectives. Two additional articles were added following reference screening. The results were 36 articles that met the inclusion criteria (See Figure I).

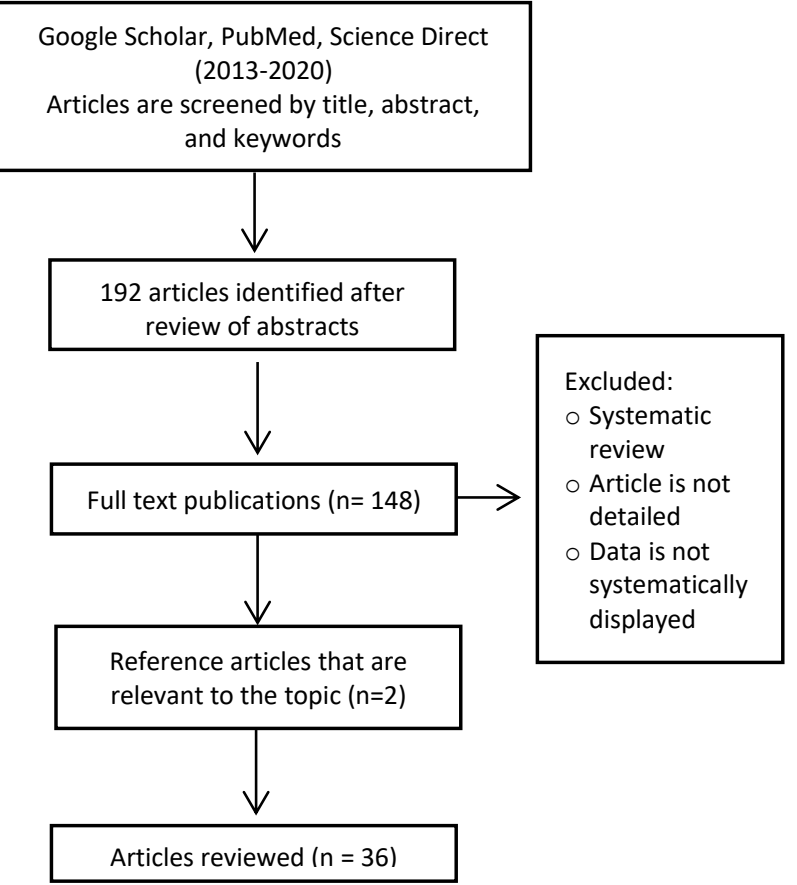




\section{Figure 1: Flow chart of articles selection}

\section{Results}

This study examined the prevalence of self-medication with short-term antibiotics from 29 countries out of a total of 48 Asian countries. Table I shows that there are 36 articles reporting on the main symptoms and the prevalence of self-medication with short-term antibiotics in Asian countries. The sample sizes ranged from 250 to 11,192 participants, and publication date ranged from 2013 to 2020; the sample population varies including the general public and other subgroup populations such as students (including medical and non-medical students); patients (attending pharmacies and other health facilities); age $\geq 18$ years. The symptom categories reported to be various, and the symptoms most often treated with antibiotics were influenza or influenza-like syndromes. Prevalence (\%) from the table means the percentage of the population that took part in self-medication with short-term antibiotics without consulting a medical doctor, i.e. using leftover antibiotics from previous treatments or getting antibiotics at the pharmacy without a prescription at a given time.

Table I: Articles reporting the prevalence of self-medication with short-term antibiotics usage in Asian countries

\begin{tabular}{|c|c|c|c|c|c|}
\hline Authors, year & Countries & $\begin{array}{l}\text { Sample } \\
\text { Size }\end{array}$ & Population & Main Symptoms & $\begin{array}{c}\text { Prevalence } \\
\%\end{array}$ \\
\hline $\begin{array}{l}\text { Barik, Islam, Kumar, } \\
\& U . \text { Haque, } 2017\end{array}$ & Bangladesh & 4,100 & Pharmacy patients & $\begin{array}{l}\text { Fever, cold \& cough, } \\
\text { dysentery, diarrhoea and } \\
\text { food poisoning, respiratory } \\
\text { tract infection, UTI, } \\
\text { toothache and viral disease }\end{array}$ & 23.5 \\
\hline Biswas et al., 2014 & Bangladesh & 1,300 & Pharmacy patients & $\begin{array}{l}\text { Dysentery, diarrhoea, food } \\
\text { poison, the common cold }\end{array}$ & 26.69 \\
\hline $\begin{array}{l}\text { Adhikari, Tshering, } \\
\text { Tshokey, Wangmo, \& } \\
\text { Wangdi, } 2017\end{array}$ & Bhutan & 692 & General public & $\begin{array}{l}\text { Common cold, any illness } \\
\text { with fever }\end{array}$ & 23.6 \\
\hline Prien, 2018 & Cambodia & 450 & $\begin{array}{l}\text { Outpatient of the } \\
\text { referral hospital }\end{array}$ & Minor illness & 49.4 \\
\hline $\begin{array}{l}\text { Li, Lin, Wang, Xuan, } \\
\text { \& Zhou, } 2018\end{array}$ & China & 11, 192 & University student & Prophylaxis & 63.1 \\
\hline Zhu et al., 2016 & China & 1,086 & University students & $\begin{array}{l}\text { Sore throat, fever, cough, } \\
\text { runny nose }\end{array}$ & 47.9 \\
\hline $\begin{array}{l}\text { Manikandan, } \\
\text { Muruganandhan, } \\
\text { Priya, Shamsudeen, } \\
\text { \& Sujatha } 2018\end{array}$ & India & & Dental clinic patients & Dental problems & 71.2 \\
\hline $\begin{array}{l}\text { Kapoor, Makhija, } \\
\text { Nair, Nandigam, \& } \\
\text { Virmani } 2017\end{array}$ & India & 531 & Health science students & $\begin{array}{l}\text { Sore throat, flu-like } \\
\text { symptoms, skin infection }\end{array}$ & 48 \\
\hline $\begin{array}{l}\text { Kurniawan, Posangi, } \\
\text { \& Rampengan, } 2017\end{array}$ & Indonesia & 400 & $\begin{array}{l}\text { Community health } \\
\text { centre }\end{array}$ & Wound and skin infections & 45 \\
\hline Widayati, 2013 & Indonesia & 559 & $\begin{array}{l}\text { Adults } \\
\text { (household's survey) }\end{array}$ & $\begin{array}{l}\text { Common-cold, cough, sore } \\
\text { throat, headache, itching, } \\
\text { toothache, fever }\end{array}$ & 58 \\
\hline $\begin{array}{l}\text { Ahmed, M, \& Ali, } \\
2019\end{array}$ & Iraq & 344 & Pharmacy students & $\begin{array}{l}\text { Headache, cough, and } \\
\text { diarrhoea }\end{array}$ & 62.79 \\
\hline $\begin{array}{l}\text { Abedi, Dehghani, } \\
\text { Emad, \& } \\
\text { Ghahramani, } 2020\end{array}$ & Iran & 1,200 & $\begin{array}{l}\text { Outpatients of university } \\
\text { dental clinics }\end{array}$ & Dental pain & 42.6 \\
\hline $\begin{array}{l}\text { Al Baz, Law, \& } \\
\text { Saadeh, } 2018\end{array}$ & Jordan & 250 & A refugee at health care & $\begin{array}{l}\text { To cure or even prevent } \\
\text { diseases }\end{array}$ & 60 \\
\hline Yerubayev, 2019 & Kazakhstan & 472 & General population & $\begin{array}{l}\text { Cold, flu, sore throat, skin } \\
\text { and wound infection, }\end{array}$ & 63 \\
\hline Aboud \& Awad, 2015 & Kuwait & 770 & General population & $\begin{array}{l}\text { Common cold, sore throat, } \\
\text { cough, genitourinary } \\
\text { infections, superficial } \\
\text { wounds }\end{array}$ & 27.5 \\
\hline $\begin{array}{l}\text { Kitikannakorn \& } \\
\text { Phonlavong, } 2018\end{array}$ & Lao PDR & 768 & General population & $\begin{array}{l}\text { Common cold, sore throat, } \\
\text { and cough. }\end{array}$ & 85 \\
\hline
\end{tabular}




\begin{tabular}{|c|c|c|c|c|c|}
\hline Authors, year & Countries & $\begin{array}{l}\text { Sample } \\
\text { Size }\end{array}$ & Population & Main Symptoms & $\begin{array}{c}\text { Prevalence } \\
\% \\
\end{array}$ \\
\hline $\begin{array}{l}\text { El-Kheir, Hanna, } \\
\text { Mansour, Jamhour \& } \\
\text { Salameh, } 2017\end{array}$ & Lebanon & 400 & General population & $\begin{array}{l}\text { Sore throat, common cold, } \\
\text { fever }\end{array}$ & 50 \\
\hline $\begin{array}{l}\text { M. Haque et al., } \\
2019\end{array}$ & Malaysia & 649 & University students & $\begin{array}{l}\text { Runny nose, nasal } \\
\text { congestion, cough, sore } \\
\text { throat, fever, } \\
\text { aches and pains, vomiting, } \\
\text { diarrhoea, skin wounds }\end{array}$ & 39.3 \\
\hline $\begin{array}{l}\text { Kumar Sah, Kumar } \\
\text { Jha, \& Kumar Shah, } \\
2016\end{array}$ & Nepal & 327 & Nursing students & $\begin{array}{l}\text { Common illness, save time } \\
\text { and money }\end{array}$ & 50 \\
\hline Shah et al., 2014 & Pakistan & 431 & $\begin{array}{l}\text { Non-medical university } \\
\text { students }\end{array}$ & $\begin{array}{l}\text { Fever, pain relief, respiratory } \\
\text { symptoms, } \\
\text { gastrointestinal problems, } \\
\text { urinary symptoms }\end{array}$ & 47.6 \\
\hline Nazir \& Azim, 2017 & Pakistan & 527 & General population & Sore throat and flu & 26 \\
\hline Gillani et al., 2017 & Pakistan & 727 & $\begin{array}{l}\text { Non-medical university } \\
\text { student }\end{array}$ & $\begin{array}{l}\text { Gastrointestinal problems, } \\
\text { pain relief, respiratory } \\
\text { symptoms, } \\
\text { fever, urinary problems }\end{array}$ & 45 \\
\hline Abu Taha et al., 2016 & Palestine & 375 & Adults & Cold, flu & 38.7 \\
\hline $\begin{array}{l}\text { Bulario, Cruz, } \\
\text { Gutierrez \& Pilapil, } \\
2018\end{array}$ & Philippines & 390 & Mothers of children $<18$ & Cough & 42.05 \\
\hline $\begin{array}{l}\text { Abdel-Rahman, } \\
\text { Aljayyousi, El- } \\
\text { Heneidy, Faisal \& } \\
\text { Kurdi, } 2019\end{array}$ & Qatar & 596 & University students & $\begin{array}{l}\text { Previous illnesses that had } \\
\text { similar symptoms }\end{array}$ & 82 \\
\hline $\begin{array}{l}\text { Alarifi, Alghadeer, } \\
\text { Alhammad, Aljuaydi, } \\
\text { \& Babelghaith, } 2018\end{array}$ & Saudi Arabia & 1,264 & General population & Tonsillitis & 34 \\
\hline $\begin{array}{l}\text { Al Rasheed et al., } \\
2016\end{array}$ & Saudi Arabia & 681 & Adults & $\begin{array}{l}\text { Cough, sore throat, common } \\
\text { colds }\end{array}$ & 78.7 \\
\hline $\begin{array}{l}\text { Abdelrahman et al., } \\
2017\end{array}$ & Saudi Arabia & 1,028 & Residents & Fever, common cold & 37.9 \\
\hline Rathish et al., 2017 & Sri Lanka & 285 & Medical students & Runny nose, flu & 39 \\
\hline $\begin{array}{l}\text { Al-kayali, \& Haroun, } \\
2017\end{array}$ & Syria & 436 & Medical students & Headache, fever, and flu & $\begin{array}{l}60.5 \\
40.9\end{array}$ \\
\hline
\end{tabular}

\begin{tabular}{|c|c|c|c|c|c|}
\hline $\begin{array}{l}\text { Havanond, } \\
\text { Hongsranagon, } \\
\text { Pannoi, \& Sirijoti, } \\
2014\end{array}$ & Thailand & 396 & Adults & Feeling unwell & 33.84 \\
\hline $\begin{array}{l}\text { Dönmez, Göv, \& } \\
\text { Güngör, } 2018\end{array}$ & Turkey & 570 & Nursing students & $\begin{array}{l}\text { Colds and common cold, } \\
\text { sore throat, } \\
\text { toothache/swelling, fever, } \\
\text { cough, stomach ache, } \\
\text { weakness, hot urine, and } \\
\text { skin infections }\end{array}$ & 31.1 \\
\hline $\begin{array}{l}\text { Abduelkarem et al., } \\
2019\end{array}$ & $\begin{array}{l}\text { United Arab } \\
\text { Emirates }\end{array}$ & 315 & General population & Sore throat, runny nose & 31.7 \\
\hline Belkina et al., 2014 & $\begin{array}{l}\text { Saudi Arabia, Yemen, } \\
\text { Uzbekistan }\end{array}$ & 1,200 & $\begin{array}{l}\text { General education } \\
\text { teachers }\end{array}$ & Cough and influenza & $\begin{array}{l}48.4 \\
78.2 \\
78.3\end{array}$ \\
\hline Ha, \& Nguyen, 2019 & Vietnam & 1,000 & $\begin{array}{l}\text { Household survey (door } \\
\text { to door survey in } \\
\text { community) }\end{array}$ & Illness symptoms & 83.3 \\
\hline $\begin{array}{l}\text { Albawani, Abd-aziz, } \\
\text { \& Hassan, } 2017\end{array}$ & Yemen & 363 & $\begin{array}{l}\text { Consumers attending } \\
\text { community pharmacies }\end{array}$ & $\begin{array}{l}\text { Cold, cough, diarrhoea, and } \\
\text { fever }\end{array}$ & 87.1 \\
\hline
\end{tabular}

The authors also investigated the factors that influence self-medication with short-term antibiotic behaviour. Table II shows that 29 countries reported the main reasons for short-term antibiotic self-medication behaviour, source of information, source of where the drugs are purchased, and choice of antibiotics. When 
exploring the data, not all of the studies the authors reviewed presented the percentage of choice of antibiotics for self-medication, and some studies did not present this data ( $\mathrm{N} / \mathrm{A}$ or data not available).

Table II: Main reasons, information from, sources, and choices of antibiotics

\begin{tabular}{|c|c|c|c|c|}
\hline Studies & Main reasons & Information from & Sources & Choices of antibiotics \\
\hline Bangladesh & $\begin{array}{l}\text { Pre-experience, reduction } \\
\text { of doctor's fees }\end{array}$ & $\begin{array}{l}\text { Advice from traditional } \\
\text { healers, own knowledge }\end{array}$ & Pharmacy & $\begin{array}{l}\text { Azithromycin (24.3\%) } \\
\text { Metronidazole (12.3\%) } \\
\text { Ciprofloxacin (12.2\%) } \\
\text { Amoxicillin (9.2\%) } \\
\text { Cefixime }(7.9 \%)\end{array}$ \\
\hline Bangladesh & $\begin{array}{l}\text { Previous medication, } \\
\text { other people's } \\
\text { suggestions }\end{array}$ & $\begin{array}{l}\text { Personal knowledge, past } \\
\text { experience }\end{array}$ & Pharmacy & $\begin{array}{l}\text { Metronidazole (50.43\%) } \\
\text { Azithromycin (20.75\%) } \\
\text { Ciprofloxacin }(11.53 \%) \\
\text { Amoxicillin }(10.37 \%) \\
\text { Tetracycline }(7.49 \%)\end{array}$ \\
\hline Bhutan & $\begin{array}{l}\text { Saving time and } \\
\text { inconvenience of waiting } \\
\text { in lines }\end{array}$ & Personal knowledge & $\begin{array}{l}\text { Shared antibiotics; stock } \\
\text { from previously used } \\
\text { drugs, }\end{array}$ & Amoxicillin \\
\hline Cambodia & $\begin{array}{l}\text { Saving time, spending less } \\
\text { money, living far from } \\
\text { health facilities }\end{array}$ & $\begin{array}{l}\text { Television, physician, } \\
\text { radio, social network/ } \\
\text { website, health care } \\
\text { staffs, pharmacist, and } \\
\text { newspaper }\end{array}$ & Pharmacy/drug store & $\mathrm{N} / \mathrm{A}$ \\
\hline China & $\begin{array}{l}\text { Success of previous } \\
\text { treatment }\end{array}$ & $\begin{array}{l}\text { Personal experience, } \\
\text { health professional }\end{array}$ & $\begin{array}{l}\text { Leftover, pharmacy, given } \\
\text { by others }\end{array}$ & N/A \\
\hline China & $\begin{array}{l}\text { Convenience, cost saving, } \\
\text { success of previous } \\
\text { treatment }\end{array}$ & $\begin{array}{l}\text { Personal experience, } \\
\text { family member, } \\
\text { pharmacist }\end{array}$ & $\begin{array}{l}\text { Pharmacy, stock of } \\
\text { previously used drugs }\end{array}$ & N/A \\
\hline India & $\begin{array}{l}\text { Success of previous } \\
\text { prescription, } \\
\text { availability of medicine, } \\
\text { long waiting line in clinics, } \\
\text { cost saving }\end{array}$ & $\begin{array}{l}\text { Previous prescription, } \\
\text { friends, family, internet, } \\
\text { advertisements }\end{array}$ & Leftover, pharmacy & N/A \\
\hline India & $\begin{array}{l}\text { Success of previous } \\
\text { prescription }\end{array}$ & Pharmacist & Pharmacy & $\begin{array}{l}\text { B-lactams } \\
\text { Fluoroquinolones } \\
\text { Macrolides Tetracyclines }\end{array}$ \\
\hline Indonesia & $\begin{array}{l}\text { More practical way than } \\
\text { seeking a doctor for a } \\
\text { treatment, too busy to } \\
\text { see a doctor, success of } \\
\text { previous treatment, } \\
\text { having not enough money } \\
\text { to pay for the doctor visit }\end{array}$ & Old prescription & Pharmacy & $\begin{array}{l}\text { Amoxycillin } 68.3 \% \\
\text { Ampicillin } 26.1 \%\end{array}$ \\
\hline Indonesia & $\begin{array}{l}\text { Success of previous } \\
\text { medication (54\%) }\end{array}$ & $\begin{array}{l}\text { Health professionals, } \\
\text { friends/relatives, and } \\
\text { drug leaflets. }\end{array}$ & $\begin{array}{l}\text { Pharmacy, } \\
\text { kiosks, } \\
\text { drug stores }\end{array}$ & $\begin{array}{l}\text { Amoxicillin Ampicillin } \\
\text { Ciprofloxacin Tetracycline }\end{array}$ \\
\hline Iraq & $\begin{array}{l}\text { Quick relief desired, } \\
\text { convenience, and } \\
\text { avoiding waiting at clinics }\end{array}$ & Personal knowledge & Pharmacy & N/A \\
\hline Iran & $\begin{array}{l}\text { Severe pain, previous self- } \\
\text { medications, and high } \\
\text { costs of dental visits }\end{array}$ & Previous prescription & $\begin{array}{l}\text { Pharmacy, } \\
\text { leftover, family/ friends }\end{array}$ & $\begin{array}{l}\text { Amoxicillin } \\
\text { Metronidazole Cefixime } \\
\text { Azithromycin Penicillin }\end{array}$ \\
\hline Jordan & $\begin{array}{l}\text { Long waiting hours of } \\
\text { seeking medical advice }\end{array}$ & Medical advice & $\begin{array}{l}\text { Pharmacy, leftover, share } \\
\text { drugs }\end{array}$ & N/A \\
\hline Kazakhstan & $\begin{array}{l}\text { Success of previous } \\
\text { medication }\end{array}$ & $\begin{array}{l}\text { Medical advice, internet, } \\
\text { friend/family }\end{array}$ & Pharmacy, leftover & N/A \\
\hline Kuwait & Speedy recovery & $\begin{array}{l}\text { Previous prescription, } \\
\text { family/friends }\end{array}$ & $\begin{array}{l}\text { Pharmacy, leftover, } \\
\text { sharing with family }\end{array}$ & N/A \\
\hline Lao PDR & Curing illness & $\begin{array}{l}\text { Health care providers, } \\
\text { relatives, television, radio } \\
\text { and internet }\end{array}$ & Pharmacy & N/A \\
\hline
\end{tabular}




\begin{tabular}{|c|c|c|c|c|}
\hline Studies & Main reasons & Information from & Sources & Choices of antibiotics \\
\hline $\begin{array}{l}\text { Lebanon, Beirut } \\
\text { Tripoli }\end{array}$ & Curing illness & Health professional & Pharmacy & N/A \\
\hline Malaysia & $\begin{array}{l}\text { Cost saving and } \\
\text { convenience }\end{array}$ & previous prescription & Pharmacy & $\begin{array}{l}\text { Penicillin Doxycycline } \\
\text { Clarithromycin }\end{array}$ \\
\hline Nepal & $\begin{array}{l}\text { Success of previous } \\
\text { medication }\end{array}$ & $\begin{array}{l}\text { Success of previous } \\
\text { medication }\end{array}$ & Pharmacy & \\
\hline Pakistan & $\begin{array}{l}\text { Saving time and money, } \\
\text { avoiding hassle of going } \\
\text { to doctor, success of } \\
\text { previous medication }\end{array}$ & $\begin{array}{l}\text { Personal knowledge, old } \\
\text { prescription }\end{array}$ & Pharmacy & $\begin{array}{l}\text { Amoxicillin }(41,4 \%) \\
\text { Metronidazole }(30.5 \%) \\
\text { Ciprofloxacin (12.7\%) }\end{array}$ \\
\hline Pakistan & $\begin{array}{l}\text { Success of previous } \\
\text { medication }\end{array}$ & $\begin{array}{l}\text { Previous experience, } \\
\text { pharmacists, } \\
\text { relatives/friends, doctors, } \\
\text { leaflets }\end{array}$ & Pharmacy, leftover & $\begin{array}{l}\text { Amoxiclav (40\%) } \\
\text { Ciprofloxacin (14\%) } \\
\text { Metronidazole (11\%) } \\
\text { Doxycycline }(10 \%) \\
\text { Azithromycin }(8 \%)\end{array}$ \\
\hline Pakistan & $\begin{array}{l}\text { Saving time and money, } \\
\text { avoiding hassle, success } \\
\text { of previous treatment }\end{array}$ & $\begin{array}{l}\text { Personal knowledge, } \\
\text { friends, parents, } \\
\text { pharmacist }\end{array}$ & Leftover, pharmacy & $\begin{array}{l}\text { Metronidazole } \\
\text { Ciprofloxacin } \\
\text { Amoxicillin } \\
\text { Co-Trimoxazole }\end{array}$ \\
\hline Palestine & $\begin{array}{l}\text { Preventing symptoms } \\
\text { from getting worse }\end{array}$ & $\begin{array}{l}\text { Doctors, pharmacists, } \\
\text { friends/ relatives, } \\
\text { websites, television. }\end{array}$ & $\begin{array}{l}\text { Pharmacy, leftover, } \\
\text { friends/ } \\
\text { relatives }\end{array}$ & N/A \\
\hline Philippines & $\begin{array}{l}\text { Success of previous } \\
\text { medication }\end{array}$ & $\begin{array}{l}\text { Health centres and other } \\
\text { sources }\end{array}$ & Pharmacy, health centres & Amoxicillin (50.25\%) \\
\hline Qatar & $\begin{array}{l}\text { Success of previous } \\
\text { medication }\end{array}$ & $\begin{array}{l}\text { Pharmacist, old } \\
\text { prescription }\end{array}$ & Pharmacy & $\mathrm{N} / \mathrm{A}$ \\
\hline Saudi Arabia & $\begin{array}{l}\text { Success of previous } \\
\text { medication }\end{array}$ & $\begin{array}{l}\text { Previous doctor's } \\
\text { prescription, } \\
\text { advertisements from } \\
\text { websites, social media, } \\
\text { TV, or reading }\end{array}$ & Pharmacy & $\begin{array}{l}\text { Amoxiclav (45.1\%) } \\
\text { Amoxicillin (39.9\%) }\end{array}$ \\
\hline Riyadh, Saudi & Curing symptoms & Friends, nearby pharmacy & Pharmacy & $\begin{array}{l}\text { Amoxicillin } \\
\text { Ciprofloxacin } \\
\text { Penicillin }\end{array}$ \\
\hline Saudi Arabia & $\begin{array}{l}\text { Getting better more } \\
\text { quickly }\end{array}$ & $\begin{array}{l}\text { Physicians, pharmacists, } \\
\text { friends/relatives }\end{array}$ & Pharmacy & N/A \\
\hline Sri Lanka & $\begin{array}{l}\text { Previous experience, } \\
\text { no access to } \\
\text { physician care }\end{array}$ & $\begin{array}{l}\text { Previous knowledge, } \\
\text { Physician, } \\
\text { pharmacist, } \\
\text { leaflet, } \\
\text { relative/friend, internet }\end{array}$ & $\begin{array}{l}\text { Pharmacy, } \\
\text { relatives/ friends, } \\
\text { households }\end{array}$ & Amoxicillin (56\%) \\
\hline Syria & $\begin{array}{l}\text { Mildness of illness and } \\
\text { time-saving }\end{array}$ & $\begin{array}{l}\text { Pharmacist, previous } \\
\text { doctor } \\
\text { prescription, } \\
\text { personal experience, } \\
\text { friends' advice }\end{array}$ & $\begin{array}{l}\text { Pharmacy, leftover of } \\
\text { previous prescription }\end{array}$ & N/A \\
\hline Thailand & $\begin{array}{l}\text { Success of previous } \\
\text { medication }\end{array}$ & $\begin{array}{l}\text { Old packaging of } \\
\text { antibiotics }\end{array}$ & Pharmacy & Amoxicillin \\
\hline Turkey & $\begin{array}{l}\text { Having no time to visit } \\
\text { the doctor, success of } \\
\text { previous medication, test } \\
\text { fees, drug store and } \\
\text { surrounding advice }\end{array}$ & Close friends and relatives & $\begin{array}{l}\text { Maintained antibiotics at } \\
\text { home, pharmacy }\end{array}$ & N/A \\
\hline UAE & $\begin{array}{l}\text { Previous experience with } \\
\text { the disease }\end{array}$ & $\begin{array}{l}\text { Previous personal } \\
\text { experience, } \\
\text { friend/relative, } \\
\text { community } \\
\text { pharmacist }\end{array}$ & Pharmacy, household. & $\begin{array}{l}\text { Penicillins } \\
\text { Macrolides } \\
\text { Quinolones } \\
\text { Cephalosporins } \\
\text { Tetracyclines }\end{array}$ \\
\hline
\end{tabular}




\begin{tabular}{|c|c|c|c|c|}
\hline Studies & Main reasons & Information from & Sources & Choices of antibiotics \\
\hline $\begin{array}{l}\text { Yemen, } \\
\text { Saudi Arabia, } \\
\text { Uzbekistan }\end{array}$ & $\begin{array}{l}\text { Poor regulation, lack of } \\
\text { access to health care, } \\
\text { cultural beliefs }\end{array}$ & $\begin{array}{l}\text { Pharmacist, friend, old } \\
\text { prescription }\end{array}$ & Pharmacy & N/A \\
\hline Vietnam & $\begin{array}{l}\text { Believing in professional } \\
\text { competences in buying } \\
\text { antibiotics at drug store } \\
\text { near home }\end{array}$ & $\begin{array}{l}\text { health professional, } \\
\text { family/friend }\end{array}$ & Pharmacy & N/A \\
\hline Yemen & $\begin{array}{l}\text { High cost of doctor } \\
\text { consultation }\end{array}$ & $\begin{array}{l}\text { Community drug } \\
\text { dispenser, family } \\
\text { member, personnel } \\
\text { choice, old prescription, } \\
\text { friends, media }\end{array}$ & Pharmacy & N/A \\
\hline
\end{tabular}

\section{Discussion}

This study shows that self-medication with short-term antibiotics behaviour is diverse in Asian countries (see Table I). Socio-demographic and economic factors have also been examined in some of these studies, providing information that the use of non-prescription antibiotics exists at all levels of socioeconomic status. However, the reasons for this behaviour, particularly those related to socio-cognitive or psychological factors, have not been explored. Therefore, more research is needed, especially the one focusing on these factors. This reinforces the importance of conducting research to develop effective strategies to stem the tide of antibiotic resistance.

\section{Prevalence}

The prevalence of self-medication with antibiotics (SMA) in this study was reported to be high, the lowest being 23.5\% in Bangladesh (U. Haque et al., 2017) and the highest being $87.1 \%$ in Yemen (Albawani et al., $2017)$, with an average of $50.8 \%$. Hence, this appears to be a health challenge in the region. This reinforces the results of health behaviour studies that are practised in most parts of the world, where more than $50 \%$ of antibiotics are purchased and used without a prescription ( Morgan et al., 2011; Högberg, Muller, Monnet \& Cars 2014; Auta et al., 2019).

The main reason for the large range of SMA prevalence may be due to differences in social, cultural, and economic status determinants. Differences are also caused by research methodology, research data collection, sample population, and time that might contribute to variations in the prevalence of SMA.

\section{Socio-demographic and socio-economic factors}

Most studies reported that the success of previous treatment influenced SMA, while other studies reported that low knowledge (Yerubayev, 2019); male gender (Emad et al., 2020); female gender (Zhu et al.,
2016); age (Zhu et al., 2016); income (Abu Taha et al., 2016) were the ones influenced SMA. Other studies also reported that the level of education (Jamhour et al., 2017) and disease severity (Al-kayali \& Haroun, 2017) were factors that were significantly related to SMA behaviour. In most studies, socio-cultural, economic, and demographic factors were reported to have an influence on the practice of self-medication with antibiotics. Older age groups were reportedly more likely to do SMA in China and Riyadh (AI Rasheed et al., 2016; Zhu et al., 2016), whereas in Tripoli (Lebanon), age did not have a significant effect (Jamhour et al., 2017). The effect of these factors is reported to be low or high depending on the research context. For example, studies in Palestine were reported to consider income with SMA practice (Abu Taha et al., 2016), while research in Yemen reported having no significant relationship with material status and monthly income (Albawani et al., 2017). Studies among people living in rural areas reported that factors, such as education level and socioeconomic status, were all related to self-medication (Phonlavong \& Kitikannakorn, 2018). The study found how selfmedicated participants had low levels of education, with almost half of them uneducated and most of them included in low socio-economic classes.

\section{Information source, drug sources, and benefits of SMA}

Information on antimicrobial agents on self-medication is obtained from a variety of sources. The majority of studies reported health workers, pharmacists, and family/friends/relatives as the main sources of information (see Table I). Other sources of information were the success of previous treatments (most studies), leaflets (Rathish et al., 2017), advertisements from websites, social media (Alghadeer et al., 2018), radio and television (Phonlavong \& Kitikannakorn, 2018). Antibiotics were obtained from various sources, including pharmacies (all studies), leftovers (most studies), relatives/friends (most studies), and other health services. The advantages of self-medication with 
antibiotics are that it is easy to get drugs at the pharmacies, saves money (Gillani et al., 2017) and time for doctor visits ( Al-kayali \& Haroun, 2017) and can cure symptoms (Al Rasheed et al., 2016). It turns out that the success of previous treatment, poor regulation, lack of access to health services, saving time or money are among the causes; similar findings are observed and reported worldwide (Lescure et al., 2018).

Information and advice about antibiotics can come from common people (uninformed people). Several studies have shown that ordinary people, especially family members, relatives, or popular friends, can become the sources of information. Informal drug providers, such as traditional medicine traders and traditional healers, who are mostly not health workers, are also popular as drug advisers, especially in developing countries. The advice given by common people is mainly based on their experience in using antibiotics that are prescribed or not. This raises questions about the suitability and appropriateness of the advice given because their previous medical conditions may differ.

Health workers are expected to be able to provide education about medicine in a professional manner (Greiner \& Knebel, 2003). The WHO states that pharmacists are health workers who have the responsibility to help people in their own treatment, including the use of antibiotics without a prescription, based on their knowledge of pharmaceutical care. Appropriate and consistent pharmaceutical services will increase the role and reputation of pharmacists in the wider community and can improve the health status of the community. In terms of time spent, pharmacists ideally prioritise providing pharmaceutical services, but there are often job conflicts as entrepreneurs who manage pharmacies with clinical pharmacy services neither reject nor question the reason for antibiotic use when requested (Hermansyah et al., 2012). They tend to delay the client's idea of the need for antibiotics. Most pharmacy employees do not have professional qualifications and know very little about pharmacies. Pharmacists trained in integrated healthcare systems have had a significant impact on minimising irrational use of antibiotics in developing countries. The increasing role of pharmacists in developing countries has the potential to have a positive impact on global AMR issues (Gajdács, Paulik, \& Szabó, 2020). Further research should be conducted to evaluate the attitudes and behaviours of healthcare practitioners towards antibiotic use.

Doctors also have a professional responsibility to provide information about medications, including the antibiotics prescribed for their patients. However, the excessive workload is often claimed as the main reason for not providing adequate information about the drugs prescribed to patients. Advice on the use of antibiotics given by common people or health workers can affect the behaviour of using antibiotics that are not prescribed in the community (Saqib et al., 2019). Therefore, this issue needs to be explored further.

\section{Misuse of self-treatment antibiotics}

The most common misuse of self-medication with antibiotics includes the inappropriate use of antibiotics for children, self-medication with antibiotics (Biswas et al., 2014), inappropriate duration of therapy of fewer than five days (Yerubayev, 2019), an incorrect indication that is used for viral infection (most studies), exchange/share of drugs (most studies), and wound healing (Kurniawan et al., 2017). The use of antimicrobials for viral infections, such as influenza and influenza-like symptoms, sore throat, and fever, was reported in nearly all studies. Other complaints reported were dysentery, diarrhoea, and food poisoning (Biswas et al., 2014; U. Haque et al., 2017). Antimicrobial agents commonly used for symptoms of viral infections included amoxicillin, ampicillin, metronidazole, azithromycin, ciprofloxacin, and tetracycline.

Several studies have shown a significant positive relationship between knowledge of antibiotics and antibiotic resistance and behaviour. Therefore, intervention studies are needed to educate the population. Because advice from medical personnel is positively related to knowledge and attitudes toward antibiotics, it is important to encourage medical staff to instruct patients. The study identified groups at high risk for poor antibiotic behaviour to increase the effectiveness of targeted interventions for reducing the abuse.

\section{Key finding}

Pharmacies are generally used as the main source of information for antibiotics that are obtained and used freely. However, community pharmacies often do not have adequate bio-medical knowledge about antimicrobial agents and disease processes. Therefore, a promotion at all levels of society is an important target for minimising SMA. The success of previous treatments tends to increase self-confidence in the ability to manage subsequent diseases without the need to consult a doctor. This is a potential risk factor for improper drug use because most patients do not have knowledge of the disease process and drugs used in self-medication. In the articles reviewed, side effects from SMA were rarely reported.

\section{Recommendation}


There is a need to develop more research (qualitative, quantitative, observational studies, prospective longitudinal studies, or retrospective studies) on factors affecting self-medication with antibiotics to optimally address public health problems. Some of the studies conducted in the majority of self-medication with antibiotics are cross-sectional, where several studies focus on knowledge, attitudes, and practices. Comprehensive qualitative research will have the benefit of increasing a deeper understanding of the phenomenon of self-medication with antibiotics. Therefore, randomised control trials (RCT) are recommended to explore the impact of self-medication with antibiotics on population groups. RCTs are essential for assessing the impact of self-medication with antibiotics on AMR to produce evidence that can guide the development of more effective health promotion strategies. Standard counselling protocols and policies regarding the prescribing and limiting of antibiotic release are important steps for controlling AMR.

\section{Limitations}

The limitations of this study are limited to the published literature, excluding potential results found in the "grey" literature.

\section{Conclusions}

Self-medication with antibiotics (SMA) is a very common practice in Asian countries. Self-medication with antibiotics is influenced by socio-cultural health factors and is often associated with poor spending and prescription practices. Self-medication with antibiotics practice is one of the most important factors contributing to the emergence of AMR, which is no longer a threat but a terrible reality.

This literature review provides an overview of the need for solutions to reduce SMA behaviour in the community, among others, by conducting pharmaceutical counselling, seminars, campaigns, pamphlets, and education through social media. Educational interventions targeting individuals and the community, in addition to increasing access to highquality public health services and enforcement of regulations on the use of drugs without prescription, can also reduce the burden of infectious diseases and help reduce the challenges of SMA.

\section{Conflict of interest}

The authors have no conflict of interest associated with the material presented in this paper.

\section{Acknowledgement}

The authors would like to thank all colleagues who have supported this research.

\section{Author contributions}

Conceptualisation: IW, SB. Data curation: IW, SB. Formal analysis: IW, SB. Funding acquisition: None. Methodology: IW, SB. Project administration: IW. Writing original draft: IW, SB. Writing, review \& editing: IW, SB, PJ, DP.

\section{References}

Abdelrahman, T.M., Saeed, M.S. Al, Karam, R.A., Alkhtami, A.M., Alswat, O. B., Alzahrani, A.A., ... Hatim A. Jawad. (2017). Misuse of antibiotics and antibiotic resistance: a public population-based health survey in Al Taif - Saudi Arabia. World Journal of Pharmaceutical and Medical Research, 2(3), 54-62

Abduelkarem, A.R., Othman, A.M., Abuelkhair, Z.M., Ghazal, M.M., Alzouobi, S.B., \& El Zowalaty, M.E. (2019). Prevalence of self-medication with antibiotics among residents in United Arab Emirates. Infection and Drug Resistance, 12, 34453453. https://doi.org/10.2147/IDR.S224720

Abu Taha, A., Abu-Zaydeh, A.H., Ardah, R.A., Al-Jabi, S.W., Sweileh, W.M., Awang, R., \& Zyoud, S.H. (2016). Public knowledge and attitudes regarding the use of antibiotics and resistance: Findings from a cross-sectional study among Palestinian adults. Zoonoses and Public Health, 63(6), 449457. https://doi.org/10.1111/zph.12249

Ahmed, F.T., M, G.Y., \& Ali, O. (2019). Evaluation of selfmedication among Iraqi pharmacy students. Journal of Ideas in Health, 2(2), 108-112. https://doi.org/10.3844/ajptsp.2012.135.140

Al Baz, M., Law, M.R., \& Saadeh, R. (2018). Antibiotics use among Palestine refugees attending UNRWA primary health care centers in Jordan - A cross-sectional study. Travel Medicine and Infectious Disease, 22, 25-29. https://doi.org/10.1016/j.tmaid.2018.02.004

Al Rasheed, A., Yagoub, U., Alkhashan, H., Abdelhay, O., Alawwad, A., Al Aboud, A., \& Al Battal, S. (2016). Prevalence and Predictors of Self-Medication with Antibiotics in Al Wazarat Health Center, Riyadh City, KSA. BioMed Research International.https://doi.org/10.1155/2016/3916874

Albawani, S.M., Hassan, Y. Bin, \& Abd-aziz, N. (2017). Selfmedication with antibiotics in Sana'a City, Yemen, 16, 11951199.

Alghadeer, S., Aljuaydi, K., Babelghaith, S., Alhammad, A., \& Alarifi, M.N. (2018). Self-medication with antibiotics in Saudi Arabia. Saudi Pharmaceutical Journal, 26(5), 719-724. https://doi.org/10.1016/j.jsps.2018.02.018

Aljayyousi, G.F., Abdel-Rahman, M.E., El- Heneidy, A., Kurdi, R., \& Faisal, E. (2019). Public practices on antibiotic use: A 
cross-sectional study among Qatar University students and their family members. PLOS ONE, 14(11), 1-22. https://doi.org/10.1371/journal.pone.0225499

Auta, A., Hadi, M.A., Oga, E., Adewuyi, E.O., Abdu-Aguye, S.N., Adeloye, D., ... Morgan, D.J. (2019). Global access to antibiotics without prescription in community pharmacies: A systematic review and meta-analysis. Journal of Infection, 78(1), 8-18. https://doi.org/10.1016/j.jinf.2018.07.001

Awad, A.I., \& Aboud, E.A. (2015). Knowledge, attitude and practice towards antibiotic use among the public in Kuwait. PLOSONE, 10(2), 1-15. https://doi.org/10.1371/journal.pone.0117910

Belkina, T., Al Warafi, A., Hussein Eltom, E., Tadjieva, N., Kubena, A., \& Vlcek, J. (2014). Antibiotic use and knowledge in the community of Yemen, Saudi Arabia, and Uzbekistan. Journal of Infection in Developing Countries, 8(4), 424-429. https://doi.org/10.3855/jidc.3866

Biswas, M., Roy, M.N., Manik, M.I.N., Hossain, M.S., Tapu, S.T.A., Moniruzzaman, M., \& Sultana, S. (2014). Self medicated antibiotics in Bangladesh: A cross-sectional health survey conducted in the Rajshahi City. BMC Public Health, 14(1), 1-7. https://doi.org/10.1186/1471-2458-14-847

Bulario, J.S., Cruz, I.L.P., Pilapil, M.C., Gutierrez, M.M. (2018). Factors Associated with Parental Self-medication of Antibiotics in Health Centers of Manila in 4th International Research Conference on Higher Education. KnE Social Sciences, 3(6), 891-910. https://doi.org/10.18502/kss.v3i6.2427

Dönmez, S., Güngör, K., \& Göv, P. (2018). Knowledge, attitude and practice of self-medication with antibiotics among nursing students. International Journal of Pharmacology, 14(1), 136-143. https://doi.org/10.3923/ijp.2018.136.143

Emad, S., Abedi, S., Dehghani, Z., \& Ghahramani, Y. (2020). Prevalence of self-medication with antibiotics amongst clients referred to outpatient university dental clinics in Iranian population: A questionnaire-based study. Iranian Endodontic Journal, 15(1), 1-5. https://doi.org/10.22037/iej.v15i1.24334

Gajdács, M., Paulik, E., \& Szabó, A. (2020). Knowledge, attitude and practice of community pharmacists regarding antibiotic use and infectious diseases: A cross-sectional survey in Hungary (KAPPhA-HU). Antibiotics, 9(2). https://doi.org/10.3390/antibiotics9020041

Gillani, A.H., Ji, W., Hussain, W., Imran, A., Chang, J., Yang, C., \& Fang, Y. (2017). Antibiotic self-medication among nonmedical university students in Punjab, Pakistan: A crosssectional survey. International Journal of Environmental Research and Public Health, 14(10), 1-9. https://doi.org/10.3390/ijerph14101152

Greiner, A.C., \& Knebel, E. (2003). Health Professions Education: A Bridge to Quality. (A. C. Greiner \& E. Knebel, Eds.), Institute of Medicine of The National Academies. Washington DC: National Academy of Sciences. https://doi.org/10.17226/10681

Ha, T. Van, Nguyen, A.M.T., \& Nguyen, H.S.T. (2019). Selfmedication practices among Vietnamese residents in highland provinces. Journal of Multidisciplinary Healthcare, 12, 493-502. https://doi.org/10.2147/JMDH.S211420

Haque, M., Rahman, N.A.A., McKimm, J., Kibria, G.M., Majumder, M.A.A., Haque, S. Z., ... Othman, N.S.A.B. (2019). Self-medication of antibiotics: Investigating practice among university students at the Malaysian National Defence
University. Infection and Drug Resistance, 12, 1333-1351. https://doi.org/10.2147/IDR.S203364

Haque, U., Kumar, A., Barik, S.M.A., \& Islam, A.U. (2017). Prevalence, Practice and Irrationality of Self-medicated Antibiotics among People in Northern and Southern Region of Bangladesh. International Journal of Research in Pharmacy and Biosciences, 4(10), 17-24.

Hardon, A., Hodgkin, C., \& Fresle, D. (2004). How to investigate the use of medicines by consumers. Amasterdam.

Haroun, M.F., \& Al-kayali, R.S. (2017). Self-medication among undergraduate medical students in two universities in Syria. International Journal of Pharmaceutical Sciences and Research, 8(4), 1881-1886. https://doi.org/10.13040/JJPSR.0975-8232.8(4).188186

Hermansyah, A., Sukorini, A.I., Setiawan, C.D., \& Priyandani, Y. (2012). The conflicts between professional and non-professional work of community pharmacists in Indonesia. Pharmacy Practice, 10(1), 33-39. https://doi.org/10.4321/s1886-36552012000100006

Högberg, L.D., Muller, A., Zorzet, A., Monnet, D.L., \& Cars, O. (2014). Antibiotic use worldwide. The Lancet Infectious Diseases, 14(12), 1179-1180. https://doi.org/10.1016/S1473-3099(14)70987-9

Jamhour, A., El-Kheir, A., Salameh, P., Hanna, P.A., \& Mansour, H. (2017). Antibiotic knowledge and selfmedication practices in a developing country: A crosssectional study. American Journal of Infection Control, 45(4), 384-388. https://doi.org/10.1016/j.ajic.2016.11.026

Kumar Sah, A., Kumar Jha, R., \& Kumar Shah, D. (2016). Selfmedication with antibiotics among nursing students of Nepal. International Journal of Pharma Sciences and Research, 7(11), 427-430. https://doi.org/10.1186/1471-2296-11-58

Kurniawan, Posangi, J., \& Rampengan, N. (2017). Association between public knowledge regarding antibiotics and selfmedication with antibiotics in Teling Atas Community Health Center, East Indonesia. Medical Journal of Indonesia, 26(1), 62-69. https://doi.org/10.13181/mji.v26i1.1589

Lescure, D., Paget, J., Schellevis, F., \& van Dijk, L. (2018). Determinants of self-medication with antibiotics in European and Anglo-Saxon countries: A systematic review of the literature. Frontiers in Public Health, 6, 1-11. https://doi.org/10.3389/fpubh.2018.00370

Morgan, D.J., Okeke, I.N., Laxminarayan, R., Perencevich, E. N., Weisenberg, S., Morgan, D.J., ... Weisenberg, S. (2011). Non-prescription antimicrobial use worldwide: A systematic review. The Lancet Infectious Diseases, 11(9), 692-701. https://doi.org/10.1016/S1473-3099(11)70054-8

Nazir, S., \& Azim, M. (2017). Assessment of antibiotic selfmedication practice among public in the northwestern region of Pakistan. European Journal of Hospital Pharmacy , 24(4), 200-203. https://doi.org/10.1136/ejhpharm-2015-000733

Nepal, G., \& Bhatta, S. (2018). Self-medication with Antibiotics in WHO Southeast Asian Region: A Systematic Review. Cureus, 10(4). https://doi.org/10.7759/cureus.2428

Phonlavong, C., \& Kitikannakorn, N. (2018). General population's knowledge, beliefs, and care-seeking behavior about antibiotics: a cross-sectional survey in Lao People's Democratic Republic. Thai Journal of Pharmacy Practice, 10(1), 412-420.

Prien, C. (2018). The awareness of antimicrobial use and antimicrobial resistance among out patients: A case study at 
Batheay referral hospital at kampong Cham Province, Cambodia. Global Health and Developlment.

Rathish, D., Wijerathne, B., Bandara, S., Piumanthi, S., Senevirathna, C., Jayasumana, C., \& Siribaddana, S. (2017). Pharmacology education and antibiotic self-medication among medical students: A cross-sectional study. BMC Research Notes, 10(1), 1-5. https://doi.org/10.1186/s13104017-2688-4

Saqib, A., Atif, M., Ikram, R., Riaz, F., Abubakar, M., \& Scahill, S. (2019). Factors affecting patients' knowledge about dispensed medicines: A Qualitative study of healthcare professionals and patients in Pakistan. PLOS ONE, 13(6), 1-22. https://doi.org/10.1371/journal.pone.0197482

Shah, S.J., Ahmad, H., Rehan, R.B., Najeeb, S., Mumtaz, M., Jilani, M.H., ... Kadir, M.M. (2014). Self-medication with antibiotics among non-medical university students of Karachi : a cross-sectional study. BMC Pharmacology and Toxicology, 15(74), 1-7.

Shamsudeen, S.M., Priya, R.S., Sujatha, G., Muruganandhan, J., \& Manikandan, K. (2018). Self medication with antibiotis: A knowledge, attitude, and practice appraisal of 610 dental pastients in Chenai, India, from 2016 to 2017. Journal of Education and Health Promotion, 7, 1-6. https://doi.org/10.4103/jehp.jehp

Singh, P. (2017). One Health approach to tackle antimicrobial resistance in South East Asia. BMJ (Clinical Research Ed.), 358, j3625. https://doi.org/10.1136/bmj.j3625

Sirijoti, K., Hongsranagon, P., Havanond, P., \& Pannoi, W. (2014). Assessment of Knowledge Attitudes and Practices Regarding Antibiotic Use in Trang Province, Thailand. J Health Res , 28(5), 299-307.

Song, J.-H. (2014). Antimicrobial Resistance Control in Asia. Monitoring, Surveillance and National Plans, 40-45. https://doi.org/10.1001/jama.2016.11764
Tshokey, T., Adhikari, D., Tshering, T., Wangmo, S., \& Wangdi, K. (2017). Assessing the knowledge, attitudes, and practices on antibiotics among the general public attending the outpatient pharmacy units of hospitals in Bhutan: A crosssectional survey. Asia-Pacific Journal of Public Health, 29(7), 580-588. https://doi.org/10.1177/1010539517734682

Virmani, S., Nandigam, M., Kapoor, B., Makhija, P., \& Nair, S. (2017). Antibiotic use among health science students in an Indian university: A cross sectional study. Clinical Epidemiology and Global Health, 5(4), 176-179. https://doi.org/10.1016/j.cegh.2017.04.005

Wang, X., Lin, L., Xuan, Z., Li, L., \& Zhou, X. (2018). Keeping antibiotics at home promotes self-medication with antibiotics among Chinese university students. International Journal of Environmental Research and Public Health, 15(4), 1-13. https://doi.org/10.3390/ijerph15040687

WHO. (2015). Anticipating Emerging Infectious Disease Epidemics. World Health Organisation..

WHO. (2016). Situational Analysis on Antimicrobial Resistance in the South-East Asia Region.

Widayati, A. (2013). Self Medication with Antibiotics in Yogyakarta City Indonesia. The University of Adelaide.

Yerubayev, Z. (2019). Knowledge, use and behavior towards antibiotics and antibiotic resistance among residents of Astana , Kazakhstan. Nazarbayev University School of Medicine.

Zhu, X., Pan, H., Yang, Z., Cui, B., Zhang, D., \& Ba-Thein, W. (2016). Self-medication practices with antibiotics among Chinese university students. Public Health, 130, 78-83. https://doi.org/10.1016/j.puhe.2015.04.005 